BLS 35, No 2 2009. DOI: http://dx.doi.org/10.3765/bls.v35i2.3511

(published by the Berkeley Linguistics Society and the Linguistic Society of America)

\title{
Argument is Gesture War: Function, Form and Prosody of Discourse Structuring Gestures in Political Argument
}

\author{
ELISABETH WEHLING \\ University of California,Berkeley
}

\section{Introduction}

Cognition is the basis of speech and gesture as co-produced and co-perceived channels of communication. McNeill (1992) shows the close conceptual linkage between gesture and speech. Sweetser (1998) notes that gestures which are not cosemantic with narrative content may function to create discourse meaning, such as managing speaker turns and manipulating speech contents as metaphorical objects. Bavelas et al. (1992) distinguish topic gestures, which refer to narrative content, from interactive gestures, which refer to the interlocutor, for the case of conversational dialogue.

The linkage between discourse-type cognition and non-narrative gestures, or, as I will call them, discourse structuring gestures (DSG, henceforth), has not yet been studied. In this paper, I look at DSG form, prosody, and function in political argument, and show that DSG in argument systemically differ from those that have been observed in conversation.

I believe that respective differences stem from the evocation of discourse type specific embodied concepts shared by interlocutors, such as primary metaphors and related frame inferential structure. While DSG in both conversation (Bavelas et al. 1992, Sweetser 1998) and argument (my data) show mappings based on the Conduit Metaphor, DSG in argument additionally display specific forms and performance modes based on the primary metaphor Argument is Physical Struggle. As for gestural function, it has been observed that non-narrative gestures in conversation may serve to "help maintain the conversation as a social system" (Bavelas et al. 1992:469), in contrast to conversation as alternating monologues. I show that the function of DSG in argument extends to discourse control over speaker role and content.

\section{Defining Discourse Structuring Gestures}

Whether we talk on the phone or to ourselves, or just go over something in our minds without speaking, we often gesture as part of our cognitive effort. My interest lies with DSG and thus gestures that occur in face-to-face communication. 


\section{Discourse Structuring Gestures in Argument}

Kendon (2004) defines gesture as any visible bodily action that creates meaning in discourse. Bavelas et al. (1992) distinguish topic and interactive gestures as the two subcategories of hand gestures.

\subsection{Gesture and Speech Co-Timing}

Topic or narrative gestures can be co-timed with speech and depict or add to narrative content. I observe that co-speech DSG frequently relate to utterances addressing the interlocutor (cf. speaker defense gestures co-timed with "Let me finish": case (6)). This accords with Bavelas et al.'s (1992) observation that paralinguistic interactive gestures have topic independent paraphrases addressing the interlocutor. Another type of co-speech DSG that I observe in my data are completely unrelated to utterance semantics. However, their co-timing with specific speech parts seems crucial for their functions: profiling or enforcing such utterance parts that are regarded crucial arguments by the speaker (cf. content offence gestures: cases (1) and (2)) and negating ideas formerly introduced by the interlocutor (cf. content defense gestures: cases (3), (4), and (5)).

\subsection{Synchronized and Complex DSG}

Gesture analyses often focus on hand gestures and Bavelas et al.'s (1992) distinction of topic and interactive gestures (Bavelas et al. 1992:469). But people gesture with many body parts: hands, head, upper body and eyebrows, to only name a few. While we may gesture with each independently, I find that many DSG are multilayered: Their meaning is a product of co-performed movement of multiple body parts. In my data, I observe two types of such gestures.

First, movement of different layers may be parallel in form and function, stemming from the same embodied concept, e.g., a speaker defense gesture (based on Speaking is Forward Movement) performed with hand and head (cf. (6:G3)) or, a content offence gesture (based on Communication is Object Transfer) performed with head and hand (cf. (2:G2)). The implementation of multiple gesture tiers seems to function to increase a gesture's pragmatic force. They frequently co-occur with strong content offence or refusal (cf. (1), (2), and (5)) or in the context of a reoccurring struggle over speaker role (cf. (6)). I will call those synchronized DSG.

Second, body parts may co-gesture but differ in form, contributing different semantics and pragmatic functions, e.g., a hand speaker defense gesture and synchronic eyebrow crunch, where the first relates to the conceptual metaphor Speaking is Forward Movement and signals: 'stop speaking' (metaphorically: stop moving), while the second is non-metaphorical and signals something along the lines of: 'I am upset by your speaking' (cf. (6:G2)). ${ }^{1}$ One may be tempted to

\footnotetext{
${ }^{1}$ Notice the different illocutionary forces of the two co-produced gestures: While the push gesture functions much like a direct directive ('stop speaking') the eyebrow crunch functions like an indirect directive ('I disapprove of your speaking'). For further discussion see Wehling: Bimodal Speech Acts, in work.
} 


\section{Elisabeth Wehling}

interpret the hand movement as carrying the main semantic weight and the eyebrow movement to be some kind of gestural intensifier. This is not the case: In the same discourse event from which the above example is drawn an eyebrow crunch is used by the gesturer to signal 'I am upset by your speaking', upon which the interviewer stops speaking as she rightly interprets the gesture as an indirect directive. I will call multilayered gestures whose layers' movements differ in form and do not relate to one single underlying cognitive concept complex DSG. They are different from synchronized DSG in that they may combine different illocutionary forces due to form differences on the multiple layers.

\subsection{Gesture Prosody}

Whether and how to parse gestural movements into those that contribute to form and those that are prosodic is to my knowledge a question that has not yet been raised in bimodality studies. One might ask why we should bother to think about form and prosody as two levels of gesture, given the vast differences between the systems 'language' and 'gesture.' The reason lies with my interest in argument specific DSG. Speech forms in argumentative discourse do not differ from words used in other dialogues. Languages have no argument specific lexicon. Aside from form frequency, the most salient characteristics of argumentative speech are prosodic: intonation, pitch, speech rhythm and so forth. I believe that the same holds for gesture, e.g., fend off and push gestures can be used in conversation and argument to signal: 'Let me finish' ${ }^{2}$ or 'I refuse what you just said'. ${ }^{3}$ While such forms are more frequent in arguments, highly salient elements of argumentative DSG are bound to be prosodic. I expect gesture prosody (or performance mode) to relate to those conceptual mappings that shape our overall discourse type cognition.

One needs to decide, then, what elements of gestural movement to consider prosodic. An eyebrow crunch co-timed with the repetition of a hand push may at first glance seem to function alike speech prosody, such as a higher pitch when repeating: "Let me finish" (cf. (6:G2)). However, the eyebrow crunch can independently establish meaning (see above). I propose to treat co-gestures that can function as autonomous form-meaning pairs as part of multilayered gestures' form. Gesture prosody, then, establishes itself on the level of performance mode: bodily intensity (speed, abruptness, force of stroke; tenseness, laxness of gesturing body part) and spatial performance (gesture radius). Increased speed and abruptness during the repetition of a hand or upper body push in fact seems to be a functional equivalent to a higher pitch and change in speech rhythm during the repetition of "Let me finish" (cf. (6:G3)).

I ought to be careful not to oversimplify the relationship between speech and gesture prosody and the complex matter of how both relate to cognition. The notions presented here are tentative. I suspect one finds interesting relationships

\footnotetext{
${ }^{2}$ Conceptual Metaphor: Speaking Is Forward Movement.

${ }^{3}$ Conceptual Metaphor: Conduit Metaphor.
} 


\section{Discourse Structuring Gestures in Argument}

between the three when closely examined, e.g., gestural radius could conceptually relate to pitch: Louder speech carries, metaphorically, further. A gesture that reaches far towards an interlocutor seems to serve the same pragmatic function.

\section{Dialogue Subtypes: Metaphorical and Frame Inferential Mappings}

Interlocutors access speech and gesture meaning via shared embodied knowledge, such as primary metaphor. Production and perception of speech and gesture frequently draw from the same cognitive structures (McNeill 1992, Sweetser 1998). Gestural mappings have been accounted for in gesture with regard to form: "In gesture (...), there are systematic metaphorical mappings (..) remarkably parallel to the mappings seen in linguistic usage" (Sweetser 1998:2).

My hypothesis for DSG is that how we gesture (form and prosody) and why we gesture in a certain way (function) relates to conceptual metaphors and frame inferences that structure our discourse type cognition. Commonalities and differences between DSG in conversation and argument are grounded in respective conceptual commonalities and differences.

Grady (1997) defines primary metaphors as based on reoccurring subjective and sensorimotor experiential correlations. An experiential scenario that gives rise to mappings onto the target domain 'dialogue' is that of object exchange. As a child, you give an object to someone so that he or she can $\operatorname{see}^{4}$ and manipulate ${ }^{5}$ it. Reddy (1979) subsumes the resulting mappings under the Conduit Metaphor: Ideas Are Objects, Words Are Containers and Communication Is Object Transfer. Examples of linguistic usage are giving and getting ideas (Johnson and Lakoff 1980). Gestural mappings have been accounted for in conversational dialogue where interlocutors use their hands as iconic containers when offering ideas or hold hands up as if to stop oncoming objects when refusing ideas (McNeill 1985, Sweetser 1998). The conceptualization of argument has its roots in the primary metaphor Argument is Physical Struggle: Before language acquisition, interpersonal conflict comes in the form of physical struggle (Lakoff and Wehling: 2006). Later, physical struggle is accompanied by words. Our understanding of what we do when we argue with one another and how we establish control in argumentative discourse thus directly relates to notions of physical control. Johnson and Lakoff (1980) observe how respective mappings structure linguistic forms. In my data, I find gestural forms and performance modes that relate systemically to the source domain 'physical struggle', namely because of their discourse function: control over who speaks and what is being said, conceptualized in terms of physical control.

\section{Data Analysis}

I distinguish two functional classes: offence and defense gestures, regarding speaker or content. Both classes are structured by Argument is Physical Struggle

\footnotetext{
${ }^{4}$ Conceptual Metaphor: Knowing Is Seeing.

${ }^{5}$ Conceptual Metaphor: Thinking Is Object Manipulation.
} 


\section{Elisabeth Wehling}

and the entailed concept Argument Control is Physical Control. Notice that this does not predict that one finds metaphorical form mappings in each gesture. Gestures' form and prosody may "just" stem from the discourse conceptualization's inferential structure and be iconic for the kind of movement one would implement when involved in an actual physical struggle.

\subsection{Content and Speaker Control Gestures}

Content control gestures are the product of a blending of Argument is Physical Struggle and Conduit Metaphor (including the frame inferential performance parameters Mode of Content Communication is Mode of Object Transfer).

Non-narrative gestures based on the Conduit Metaphor are commonly used in conversation to 'offer' or 'refuse' ideas (Bavelas et al. 1992, Sweetser 1998). Their prototypical form is an outstretched hand with an upward facing palm (offering ideas) and a raised hand with the palm facing the interlocutor (refusing ideas). I observe those gestures in argument. This does not surprise since both conversation and argument are subtypes of 'dialogue' and respective metaphorical mappings are inherited. However, the domain 'argument' has additional frame structure that is not part of general dialogue conceptualization. What interest me are cases where argumentative content gestures differ in form and prosody from those in conversation, based on their pragmatic function.

As a result of the blending of the struggle-frame and Conduit Metaphor, ideas are conceptualized as harmful objects, as means to gain discourse control. First, metaphorical objects are not simply offered. They are pushed towards the interlocutor with the intention to 'harm', or 'hit with increased force' (cf. (1:G) and (2:G1/G2)). I call those Content Offence Gestures (COG, henceforth). Second, ideas presented by the interlocutor - conceptualized as approaching harmful objects - are in the majority of cases not just fended off, but rather pushed back. In the most graphic case by using the whole body to push an idea all the way back into the interlocutor's personal space (cf. (5)). I call those Content Defense Gestures (CDG, henceforth).

Speaker control gestures are structured by Speaking is Forward Movement and Argumentative Engagement is Physical Engagement (including frame inferential mappings onto the performance mode Mode of Speaking is Mode of Movement).

Non-narrative gestures based on Speaking is Forward Movement, such as a raised hand to signal 'stop speaking', have been observed in discourse (Kendon 1995). Such gestures used in an argument seem to differ in form and prosody from those in conversation, based on their discourse function: In argument, the kind of movement that leads to physical control is mapped onto the mode of

gesturing that leads to discourse control. First, speakers are in the majority of cases not just fended off (as is the case in less argumentative dialogue) but pushed back (cf. (6:G2,G3)). Second, the physical intensity of the gestures and their radius seem to increase in correlation with an intensified struggle over speaker role (cf. (6)). I call those Speaker Defense Gestures (SDG, henceforth). 


\section{Discourse Structuring Gestures in Argument}

Boundaries between conversational and argumentative speaker defense gestures are gradient. Form and prosody differences may (i) be a result of a shift towards a more argumentative discourse or (ii) work towards bringing about that shift. The second kind of gestures that stand in conceptual relation to Speaking is Forward Movement and Argumentative Engagement is Physical Engagement are those that accompany verbal attack: An argumentative attack on the speech tier occurs co-timed with an iconic gestural attack (cf. (7)). They are different from COG in that they are co-produced with a whole utterance, not single ideas as concrete entities. They are also different in form in that they are not iconic object pushes but rather sharp points, or 'stabs'. I call those Speaker Offence Gestures (SOG, henceforth).

\subsubsection{Content Offence Gestures}

Below, I report three instances of COG: multilayered co-speech pushes (form) executed in a sudden and forceful manner with tense gesturing body parts (prosody). All three are synchronized DSG as defined above. Two are performed with upper body and head (1:G and 2:G1), one with head and hand (2:G2).

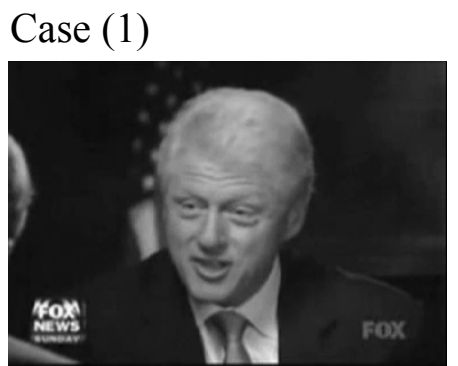

starting position

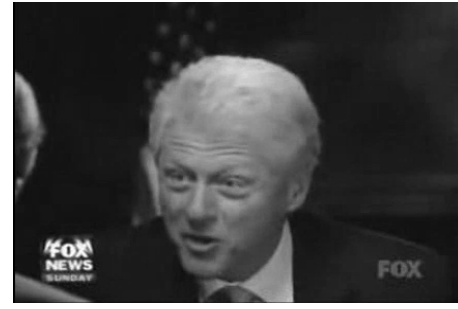

G: who (...) demoted

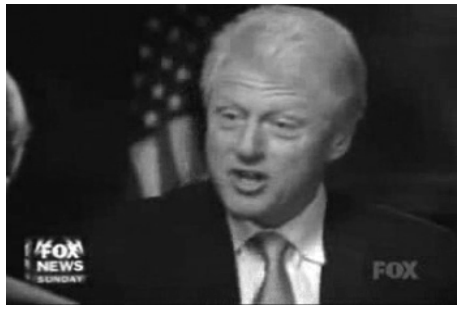

end position

Utterance tier: And the best guy in the country, Dick Clarke, who got demoted. Gesture tier: upper body / head push

Case (2)

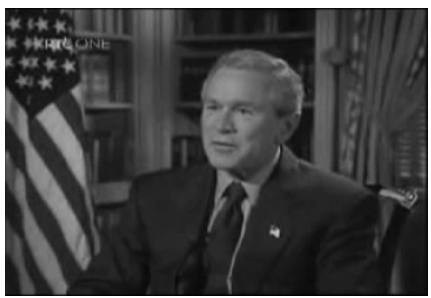

starting position

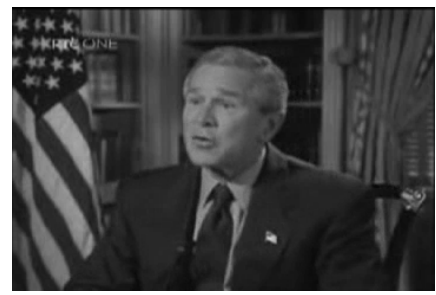

G1: against (...) people

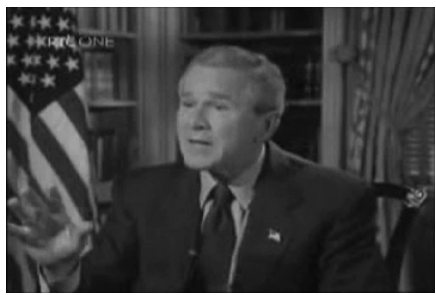

G2: against (...)-hood

Utterance tier: Saddam Hussein had weapons, used weapons of mass destruction, against his own people (G1) against the neighbourhood (G2).

Gesture tier: (G1) upper body / head push, lean back to semi-neutral position (G2) right hand / head push, palm facing interlocutor, lean back to neutral position 


\section{Elisabeth Wehling}

Why should one classify these gestures as COG? The strokes are co-timed, but not co-semantic, with a specific utterance part, namely one that is regarded a strong argument by the speaker. How do we know, aside from the gestural cue? The co-timed speech has prosodic features distinguishing it from the preceding utterance. It is produced with a rising intonation and higher pitch $(2: \mathrm{G} 1)$ and higher pitch with slower, punctuated speech rhythm (1:G). In terms of frame inferential reasoning, the gestures seem to be conceptualized as functioning to push forth crucial ideas (metaphorically: harmful objects) with the intention to weaken the interlocutor's argumentative position (metaphorically: harm by hitting with increased force).

$(1: G),(2: G 1)$ and $(2: G 2)$ show the same gestural mappings but implement different body parts $((1: \mathrm{G})$ : upper body, head; $(2: \mathrm{G} 1)$ : upper body, head; $(2: \mathrm{G} 2)$ : hand, head). In (2:G2), notice the orientation of the gesturer's hand with regard to spatial conceptualization: The palm is directed towards the interlocutor at a height and with an orientation so that, if we were to trace the line of a released forward moving object, the path endpoint would be the interlocutor's body, not some point within the shared gesture space. This is expected given argument-frame inferential mappings; the object ought to hit the interlocutor, not the ground.

How can we distinguish the above COG from CDG, SDG and SOG? The gesturer does not negate an idea prior introduced by the interlocutor, so this is not a CDG. The gesturer's speaker role is unchallenged, so this is not a SDG. The pushes are executed in a quick manner at the boundary of the gesturer's personal space. Retreat to (semi-)neutral position is part of the stroke. This makes sense in terms of the motor movement we implement when pushing forth objects. The cotimed speech parts (cf. who got demoted; against his own people; against the neighbourhood) seem to be conceptualized as concrete entities, they are part of but distinguishable from the overall utterance.

How do the gestures relate to embodied knowledge? If we push something we do it (i) from a distance without making body contact (the pushes are performed at the gesturer's space boundary; there is no metonymic physical engagement via gesture space intrusion as is the case for SOG) and (ii) via a quick and forceful forth-back movement that follows the prototypical motor routine for pushing.

\subsubsection{Content Defense Gestures}

I will report three cases of CDG. The first is mono-layered and performed with the hand (3). The second is complex where the hand gesture shows a mapping based on Ideas Are Objects and the head and facial gestures signal something along the lines of 'careful now' (4). The third is synchronized where upper body, hand and head gesture show mappings based on the Conduit Metaphor (5). CDG are co-timed with the gesturer's negation of an idea prior explicitly or implicitly introduced by the interlocutor. 
Case (3)

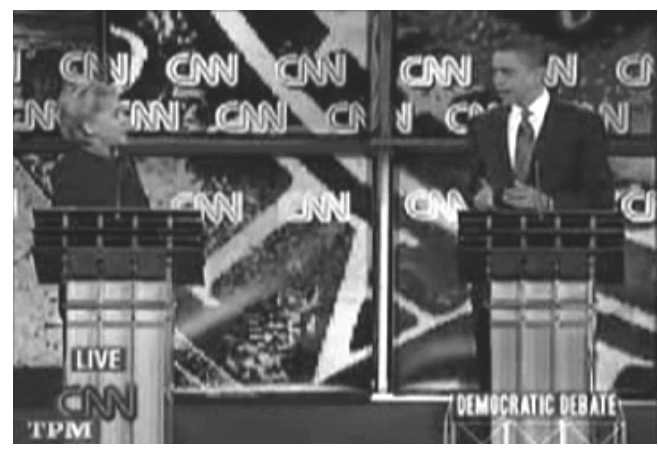

starting position

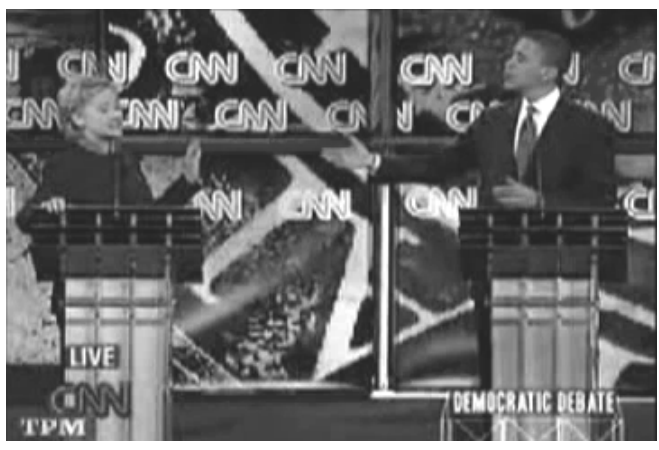

G: I (...) Reagan!

Utterance tier: Obama: Now, lets talk about Ronald Reagan. What you repeated here today is Clinton: No, nah, I did not... Obama: Wait, no, Hillary, you just spoke, you just spoke for two minutes! Clinton: $\underline{I}$ did not say anything about Ronal Reagan!

Gesture tier: left hand raise, palm towards interlocutor

Case (4)

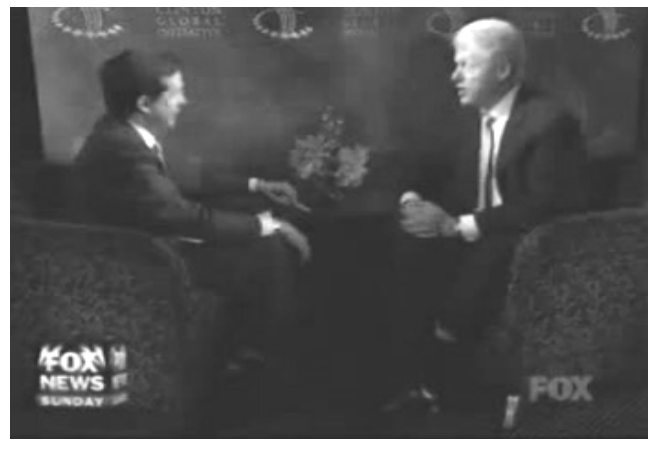

starting position

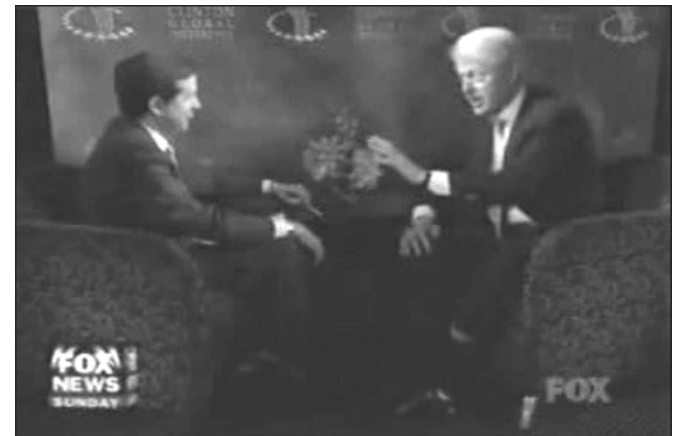

$\mathrm{G}: \underline{\mathrm{He}(. .) \text { now. }}$

Utterance tier: Clinton: Do you think Richard Clarke has a vigorous attitude about Bin Laden? Wallace: Yes, I do. I think he has a variety of opinions and loyalties, but yes. Clinton: He has a variety of opinions and loyalties now.

Gesture tier: right hand raise, head / hand forward downward push

In case (3), Barack Obama introduces the notion that Hillary Clinton brought up the topic of Ronald Reagan: "Now, lets talk about Ronald Reagan (...)". Clinton's negation "I did not say anything about Ronal Reagan" is co-timed with the hand fend off gesture. In case (4), Chris Wallace states that Dick Clarke has "a variety of opinions and loyalties". On the speech tier, Bill Clinton negates: "He has a variety of opinions and loyalties now". The gesture, a head and hand forward downward push towards Wallace, ends in co-timing with now after which Clinton retreats from Wallace's gesture space boundary. 


\section{Elisabeth Wehling}

\subsubsection{The Prosody of Content Defense Gestures}

While argumentative COG and conversational content offering gestures differ in prototypical form (push versus outstretched hand with upwards facing palm), argumentative CDG and conversational content refusal gestures are very similar in prototypical form, e.g., a raised hand facing the interlocutor, often followed by a, however slight, push. The (gradient) difference between such gestures in conversation and argument lies with prosody, especially bodily intensity and radius, e.g., in (4), Clinton's gesture is performed with a very tense hand, overall tense body posture and closeness to the interviewer's gesture space that is remarkable. He leans in specifically for the gesture. Case (5) further illustrates the linkage between discourse conceptualization and content refusal gesture prosody.

\section{Case (5)}

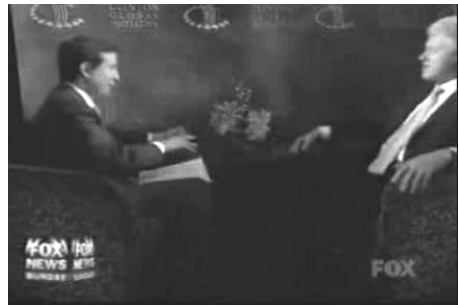

starting position

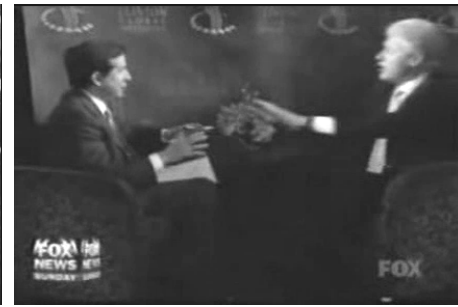

$\mathrm{G}:$ No, (...)

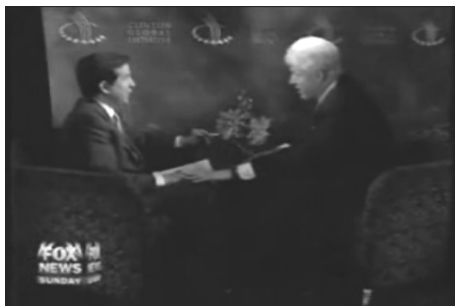

up!

Utterance tier: Wallace: With respect, if I may. Instead of going through '93 and Clinton: No, no, you asked it, you brought it up, you brought it up!

Gesture tier: Upper body forward shift, hands raised, palms facing interlocutor, hands brought downwards in interlocutor's gesture space, left hand touches knee, synchronic head push

In (5), Wallace aims to change topic: “Instead of going through '93..." implying that Clinton brought up that topic. Clinton refuses the notion: "No, no, you asked it, you brought it up". The co-timed gesture shows the strongest case of content defense we have seen so far. Clinton shifts his upper body towards the interlocutor. His hand push goes into Wallace's personal space and culminates in body contact. In terms of gestural mapping, Clinton not only fends off an oncoming object or pushes it back from a distance, he brings it all the way back to the interlocutor - as far away from his own body as possible.

Thus, content refusal force seems to correlate with both CDG form (cf. fend off: (3) versus push: (4)/(5)) and CDG prosody (general tenseness of all the strokes, and: gesture radius, compare (4)/(5)). Notice that in (3), Clinton steps into the shared gesture space to perform the gesture. In (4)/(5), Clinton leans towards Wallace (to different degrees) when performing the stroke. In terms of embodied knowledge, in an argument conceptualized as physical struggle, content refusal is stronger than in conversational dialogue since ideas put forth by interlocutors are metaphorically 'harmful' objects, a mean to gain discourse control by weakening 


\section{Discourse Structuring Gestures in Argument}

the interlocutor's position. Thus, potentially very crucial ideas (metaphorically: very harmful objects) are refused (metaphorically: fended off or pushed back) much more strongly and often as far away as possible from one's body.

\subsubsection{Speaker Defense Gestures}

I report three instances of SDG. (6:G1) is mono-layered. $(6: \mathrm{G} 2)$ is a complex gesture, namely a hand push (based on Speaking is Forward Movement), the eyebrow crunch signals: 'I am upset/angry'. (6:G3) is a synchronized gesture, a hand, head and upper body push (based on Speaking is Forward Movement).

Case (6)

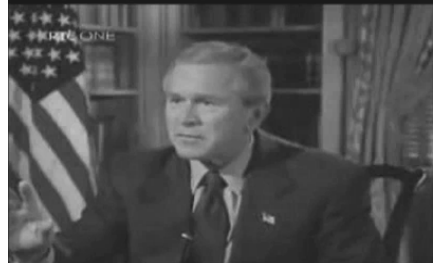

G1: May (...) finish?

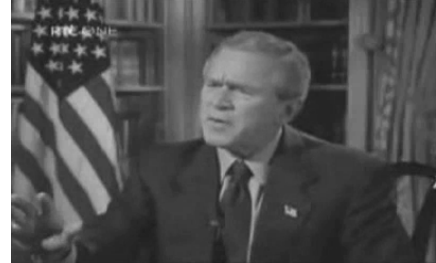

G2: Let (...) please.

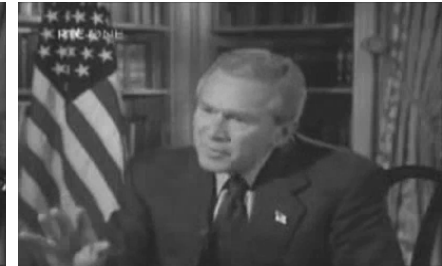

G3: Let (...) please.

Utterance tier: Bush: (...) Saddam Hussein... Interviewer: Indeed Mister President, but you didn't find... Bush: Let me, let me... May I finish? (G1) (...) Bush: It was a relative calm... Interviewer: But in your response to Iraq... Bush: Let me, let me finish, please. (G2) (...) Bush: (...) nobody cares more about the death than I do. Interviewer: Is there a point at which... Bush: Let me, let me finish.

Gesture tier: (G1) right hand raised, palm facing interlocutor

(G2) right hand raise, forward downward push, eyebrow crunch

(G3) right hand raise, forward downward push, increase in force and speed, abrupt and fragmented mode, upper body inwards downward lean

In all instances Bush is being interrupted by the interviewer. The gestures are cotimed with either direct directives (G2,G3: "Let me finish") or an indirect directive (G1: "May I finish"). The gestures enforce the speech act. Notice that while the utterance is non-metaphorical, the gesture is based on Speaking is Forward Movement. ${ }^{6}$ These are SDG, not CDG: First, discourse context and speech act clearly indicate a struggle over speaker role, and second, there is no content negation. G1 through G3 occur in sequential order. Interestingly, gesture form and prosody change in correlation with utterance politeness parameters (indirect to

\footnotetext{
${ }^{6}$ Although not reported in this paper, speech and gesture form may share gestural mapping, e.g.: The above gesture co-performed with "Stop right there", where "there" indicates a mapping based on the conceptualization of speaking as spatial movement.
} 


\section{Elisabeth Wehling}

direct directive) and prosody (increase in pitch, more segmented speech rhythm). Thus, Bush's directive gains intensity by changing speech form and prosody as well as by implementing increasingly 'strong' SDG: G1 is a mere mono-layered fend off performed with little tenseness and small radius. G2 is a complex SDG, where the push is performed with a tense hand and increased radius; the eyebrow crunch signals strong disapproval. G3 is a complex SDG where hand, upper body and head perform pushes. Notice that we can easily imagine G1 in a conversational setting, while G2 and G3 go hand in hand with a shift to a more argumentative dialogue. In terms of embodied knowledge, in an argument conceptualized as physical struggle, do we merely fend off an oncoming attacker or do we actually decide to push him or her back and if so, then how forcefully and how far from our own body?

\subsubsection{Speaker Offence Gestures}

I introduce one instance of SOG: A mono-layered right hand sharp pointing gesture, or 'stab':

Case (7)

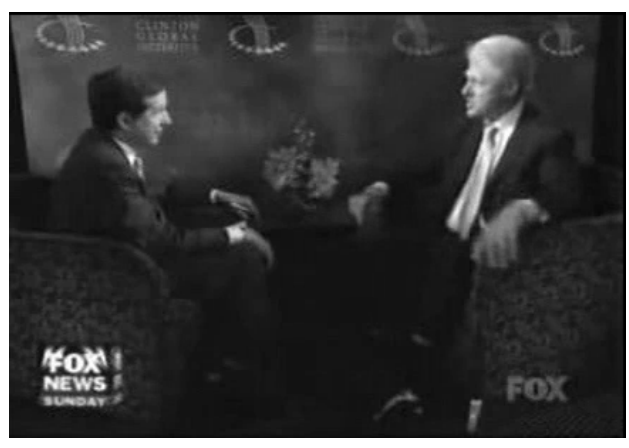

starting position

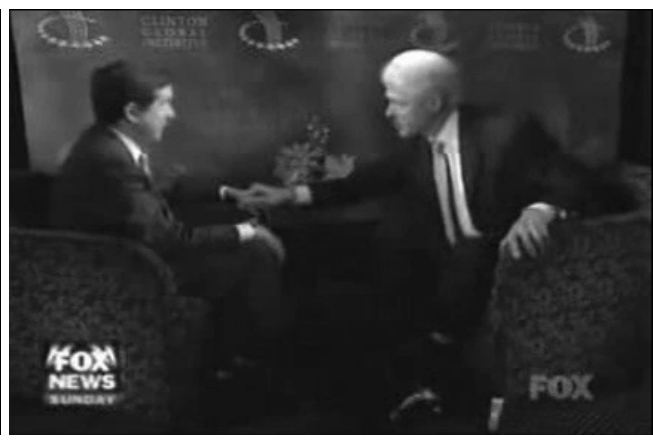

$\mathrm{G}: \underline{\text { I wanna know (...) do anything }}$

Utterance tier: I wanna know how many people (G1) in the Bush-Administration (stab1) you ask why (stab2) didn't you (stab3) do anything (stab4)

Gesture tier: hand reach towards and into interlocutor's personal space, four stabs on sheet with right hand index

In (7), the gesture refers to the question sheet between Wallace's knees, standing metonymically for questions being asked when interviewing members of the Bush administration: "I wanna know how many people (...) you ask (...)". But this is more than just a referential pointing gesture. Clinton 'stabs' onto the question sheet, several times. There is movement into the interlocutor's personal space and physical contact within that space co-timed with a whole utterance.

Referential pointing is common in conversational dialogue, e.g. to cite the interlocutor (cf. Bavelas et al.: 1992). In argumentative SOG, the interlocutor's personal space is intruded (prosody) and the gestures are classifiable as 'stabs' rather than pointing (form). In terms of embodied knowledge, the argumentative attack is accompanied and enforced by an iconic gestural attack. The inter- 


\section{Discourse Structuring Gestures in Argument}

viewer's gestural response sheds further light onto the inferential structures evoked as part of the shared discourse conceptualization: Defense from speech (and gesture) attack is physical defense, here in the form of a disengaged body posture and hand inwards shield gesture.

\section{Conclusion}

I have taken a closer look at the discourse function, form and prosody of nonnarrative gestures in relation to discourse cognition and the conceptualization of argument as physical struggle. I introduced a tentative distinction between gesture form and prosody for the analysis of DSG and argued that their specificities are a product of their discourse function, namely control over content and speaker role.

\section{References}

Bavelas, Janet Beavin, Nicole Chovil, Douglas A. Lavrie and Allan Wade. 1992. Interactive gestures. Discourse Processes 15(4):469-489.

Grady, Joseph. 1997. Foundations of meaning: Primary metaphors and primary scenes. Ph.D. diss., University of California, Berkeley.

Johnson, Mark, and George Lakoff. 1980. Metaphors we live by. Chicago: University of Chicago Press.

Kendon, Adam. 1995. Gestures as illocutionary and discourse markers in Sothern Italian conversation. Journal of Pragmatics 23(3):247-279.

Kendon, Adam. 2004. Gesture: Visible action as utterance. Cambridge: Cambridge University Press.

Lakoff, George, and Elisabeth Wehling. 2006. Auf leisen Sohlen ins Gehirn. Heidelberg: Carl-Auer Verlag.

McNeill, David. 1992. Hand and mind: What gestures reveal about thought. Chicago: University of Chicago Press.

Reddy, Michael. 1979. The conduit metaphor: A case of frame conflict in our language about language. In Andrew Ortony, ed., Metaphor and thought, 284-324. Cambridge: Cambridge University Press.

Searle, John R. 1969. Speech acts: An essay in the philosophy of language. Cambridge: Cambridge University Press.

Sweetser, Eve. 1998. Regular metaphoricity in gesture: Bodily-based models of speech interaction. In Actes du $16^{e}$ Congrès International des Linguistes [CD-ROM]. Oxford: Elsevier.

Elisabeth Wehling

Department of Linguistics

1203 Dwinelle Hall

Berkeley, CA 94720-2650

elisabethwehling@berkeley.edu 